Review Article

\title{
Techniques to analyze the effects of Radiation therapy on Enamel and Dentin - A Review
}

\author{
M ithra N. Hegde ${ }^{1}$, Nidharsh D. Hegde ${ }^{2}$, Suchetha Kumari N. ${ }^{3}$, Ganesh Sanjeev ${ }^{4}$, Priya G. , \\ Shruthi Attavar ${ }^{6}$ \\ ${ }^{1}$ Professor \& HOD, Department of Conservative dentistry \& Endodontics, ${ }^{2}$ Professor, Department of Oral and M axillofacial \\ Surgery, ABSM IDS, ${ }^{3}$ Professor, Department of Biochemistry, KSHEM A, ${ }^{4}$ Professor, Department of Radiophysics, Microtron \\ Center, M angalore University, ${ }^{5}$ unior Research Fellow, Nitte University, ${ }^{6}$ Lecturer, Department of Conservative dentistry \& \\ Endodontics, ABSM IDS.
}

*Corresponding Author : Mithra N. Hegde, Vice Principal and Dean of Dental Faculty, Head, Dept. of Conservative Dentistry \& Endodontics, A.B. Shetty Memorial Institute of Dental Sciences, Nitte University, Mangalore- 575018 M ob: +91 9845284411, E-mail: hod.cons.absmids@nitte.edu.in, drhegdedentist@gmail.com

Received : 04.04.2016

Review Completed : 11.10 .2016

Accepted

:05.11.2016

Keywords : Radiation caries, Teeth, Mechanical properties, Morphological properties

\begin{tabular}{|c|}
\hline Access this article online \\
\hline Quick Response Code \\
\hline
\end{tabular}

\begin{abstract}
Radiation therapy is a most common source in the treatment of Head and neck cancers. The therapy has a positive outcome in curing patients out of danger at an effective rate. But there are few major and minor side effects of the treatment which will possibly hinder the quality of life. One such major concern followed with the therapy is Radiation related caries. Radiation is known to damage the tissues of the teeth making it more susceptible for caries. Hence this review article details on techniques that is being used to test the mechanical and morphological properties of the teeth. The changes observed in these tissues after radiation treatment will enable us to understand the causes for caries. therefore further research in this field is necessary to develop strategies that will probably prevent radiation caries in future days.
\end{abstract}

\section{Introduction}

Cancer is one of the major health problems being faced in the world. Radiotherapy is often used as a palliative treatment for malignancies, since this method is capable of destroying tumor cells using ionizing radiation. Patients with Head and Neck Cancer (HNC) are usually accounted to high doses of radiation, where the tissues of oral cavity, maxilla, mandible and salivary glands are being exposed. Though radiotherapy preserves the tissue structure can cause adverse reactions in the oral cavity. ${ }^{1-2}$ The severity of these reactions depends on the volume, radiation location, total dose, fractionation, age, clinical conditions of the patient and associated treatments. These reactions can occur in the acute phase (during or within the first weeks of treatment) or in the chronic phase (months or years after the radiotherapy), which may include the conditions such as mucositis, candidiasis, taste alteration, osteoradionecrosis, soft tissue necrosis, dry mouth and radiation caries. ${ }^{3.5}$ Hence gaining knowledge on techniques to analyze the effects of Radiation therapy on Enamel and Dentin is of paramount importance which will detail on alterations in the mechanical and morphological properties of tissues after radiation treatment. These data can be correlated to the clinical scenario leading to the radiation caries. This analysis also helps us to understand the etiopathology of the disease and enable to design preventive measures for radiation caries accordingly.

\section{Objective}

Analysis of the techniques used to study the effect of radiation therapy on tooth Enamel and Dentin. 


\section{Methodology}

The content included in this paper is reviewed based on the articles obtained from the Pubmed, Medknow and open access journals. Initial search was on Radiation effects and Oral Health, that secured 10,000 and above related articles. Then the search was made concise by applying filters to select those articles from the year 1990 onwards. After filtering the search with keywords such as mechanical properties of the teeth, morphological properties, techniques to analyse properties of teeth and oral health guidelines, total of 53 papers were selected for our review.

\section{Discussion \\ Radiation therapy}

Radiotherapy uses high-energy rays to wither the tumors, thus killing cancer cells. ${ }^{6}$ The radiation types used for the treatment are $\mathrm{X}$ rays, gamma rays, and charged electrons or protons. In general there are 3 modes in clinical radiation stratergies:

1. Radiation can be subjected outside the human body by an instrument (external-beam radiation therapy),

2. A radioactive substance can be placed inside the body in the vicinity of cancerous cells (internal radiation therapy, also called brachy therapy).

3. A radioactive substance can be used which traverse in the blood killing cancerous tissues eg: radioactive iodine, (Systemic radiation therapy).

Every treatment modality performs to increase the fatality rate of cancerous cells by destructing the components of DNA . ${ }^{6}$ This DNA damage can occur in 2 events, by direct effect on DNA causing to lose its integrity, and by the indirect effect that will enable cells to generate free radicals which will eventually damage DNA.

\section{Radiation Dose}

Radiation dose for Head and Neck Cancer patients depends on the tumor size and for early stages it varies from 66- 74 Gy (2.0 Gy/ fraction; daily in 7 week). Concurrent chemoradiation therapy is the current treatment care regimen, where the fractionation dose is of $\geq 70 \mathrm{~Gy}$ (2.0 Gy/ Fraction). In cases of post-operative radiation treatment, the doses range from 60-66Gy (2.0 Gy/fraction, $\geq 6$ weeks). ${ }^{7}$

\section{Manifestations in the Oral Cavity}

Most of the HNC patients experiences some of the very common issues in the oral cavity which could be manageable and can also become serious in which the treatment must be terminated. M ost common oral cavity deliberations that occur after chemo-radiotherapy are mucositis (inflammation in the mucous membrane), infection, pain and bleeding. Radiations cause xerostomia damaging the salivary glands and trismus where it damages the muscles and joints of jaw and neck. they can also lead to hypo-vascularization of the bones of the maxilla or mandible due to reduction of supply of blood. In addition to these they can also cause caries, soft tissue necrosis and osteonecrosis.

Oral mucositis and xerostomia are the common complications among the HNC patients with squamous cell carcinoma, who are being subjected to radiation therapy at the oropharyngeal regions. ${ }^{9 \cdot 10 .}$ The xerostomia develops at the initial stages of radiotherapy and becomes more prominent after 20Gy dosages by thickening of the saliva. ${ }^{11}$ These changes in saliva affects its flow rate, volume and viscosity, which is due to the damage occurred in salivary glands caused by radiation. The salivary gland recovery depends on patient health and percentage of irradiation. ${ }^{12}$ though the condition is usually irreversible, there is recovery rate of up to $50 \%$, if the glands are protected and exposed to the minimum dosage of radiation.

\section{Radiation effects on Teeth}

The distinguishing features of mineralized and nonmineralized tissues of the tooth have provided them with remarkable physical properties. The hardest mineralized tissue known as Enamel forms the anatomic crown and is supported by a hard connective tissue Dentin, which is avascular and also extends elasticity to the tooth structure. The dentin is followed by the root connecting to the cementum, a mineralized tissues composed of inorganic matter same as that of the bone. In addition, there also forms Pulp-Dentin complex, where a soft connective tissue supports Dentin to maintain its rigid nature. ${ }^{15-16}$ 
This dynamic structural complexity of teeth can undergo detoriation due to prolonging effects of Radiation therapy. It can cause both short-term and long-term effects later to post treatment. Though radiation therapist's work on minimizing the side effects, side effects on the teeth and tissues of oral cavity has not gained control. ${ }^{17}$

\section{Problems with Tooth Development}

As studied by the researchers, it is observed that children who received radiation to the head and neck region were associated with the problems leading to the tooth development. The cause being that radiation had affected both the deciduous and permanent teeth. The possible reasons were the limitation in the formation of teeth enamel, shortening of the roots leading to fall out of teeth or the emergence of permanent teeth through the gums did not occur at the specific time of growth at the right position ${ }^{17 .}$ These short comings during the development period will longer the process and misplace the alignment of teeth.

\section{Radiation Caries}

Radiation-related caries is one of the late indirect effects of radiation seen among the HNC patients. It is a highly complex and destructive disease causing serious problems in the tooth cavity. The loss in the structural stability of tooth enamel and dentin due to ionizing rays is the proven evident for its causes. But the reversion rate of tooth components is minimal to prevent the future consequences of post radiation caries, thus having a negative impact on the life of HNC patients ${ }^{18 .}$

\section{Effect on Tissue Structure}

The unusual properties of Enamel and Dentin undergo changes while subjected to ionizing radiation. The crystalline nature of these dental tissues lose its structural identity when subjected to radiation, resulting in variations that alters the microhardness property, weakening of dentino-enamel junction and also causing acid solubilisation of the enamel. Alteration in the mechanical properties of teeth and changes in the enamel surfaces are eventually leading to formation of caries.
In general, the microstructure of the Enamel is generated due to interactions between enamel proteins and minerals. During enamel maturation, a part of these extracellular matrix proteins gets degraded and the left out proteins combines with the apatite crystals to form a network of organic material. This maintains the enamel toughness and prevents caries development. The crystal alignment creates structures like rods known as enamel prisms, separated by an inter-rod substance consisting of apatite crystallites. ${ }^{15-16}$

Some of the studies have reported that the apatite crystals incorporate sodium, carbonate, and magnesium by entrapment during their formation. Irradiation can cause the tissue lose its structural stability and undergo mobilization, removing the entrapped ions. This occurs due to a higher frequency in enamel that contains most of the inorganic matter as compared to dentin ${ }^{27 .}$ The combined modification of morphological features, composition and metabolism rate among intra- and inter-tubular collagen molecules causes bond strength variations in dentin. ${ }^{28}$ But major radiotherapy variations are seen in dentin due to damage to collagen fibrils resulting in a significant reduction in hardness and instability of the dentinoenameljunction. ${ }^{29}$

\section{Techniques to analyze the changes in mechanical properties of teeth after radiation \\ Hardness test}

The change in the mechanical properties of the material can induce change in the microstructural arrangement of its elements. The one of the best method employed for studying mechanical property is testing for its micro hardness property. The M icro hardness value of a material can be measured by the depth of an indentation mark left by an indenter against a specific force per time. There are four common methods for expressing the hardness of a material: Brinell, Rockwell, Vickers, and Knop. Each of these methods varies with its applications.

The Brinell hardness test is the oldest method used to measure the surface hardness of metals and alloys. It consists of an indenter made up of hardened steel or 
carbide ball of $10 \mathrm{~mm}$ in diameter.

The Rockwell hardness test method evaluates the surface hardness of the plastic materials used in dentistry. The material is tested using an indenter made up of a diamond cone or hardened steel ball.

Vickers test is used to test the hardness of minute areas and of very hard materials. It consists of a diamond indenter, pyramid shaped with a square base. While testing, the load is normally applied for 10 to 15 seconds. The indentation mark left in the surface of the material after removal of the load is measured using a microscope and their average is calculated.

Knoop Hardness is more sensitive method used to study the surface characteristics of the material. It is a diamond indenter ground to pyramidal form and it gives a diamond shaped indentation mark on the surface of the material.

M icro Hardness tests are extensively used and have wide applications in Dentistry. An appropriate force applied against specific time and distance will give data to interpret the hardness of the material. They have been widely used to evaluate the degree of mineralization of a dental substrate. As studied in previous section, the tooth loses its tissue integrity after radiation treatment, thereby undergoing demineralization of tooth enamel and dentin. Hence assessment of enamel and dentin remineralization and demineralization after different treatments is of utmost importance to study the mechanical property of the tissues. Other important use of M icro hardness testing in dentistry is to evaluate the degree of polymerization of composites, cements and resins. The most commonly employed hardness technique is Vickers and Knoop test to determine the hardening and depth of cure of dental materials.

The effect of radiation in damaging the enamel and dentin resulting in caries is extensively studied by the researchers all over the world. Accordingly most of them have concluded in their studies that ionizing rays has no much effect on the enamel micro hardness of permanent teeth but micro hardness had increased in the superficial enamel region. Meanwhile the micro hardness of dentin had abruptly decreased after treatment with the greatest reduction in the middle dentin -region. ${ }^{29-30}$ These variations caused by radiation makes the enamel brittle which are more susceptible to breakage. Some limited studies have shown to have decrease in Enamel microhardness with subsequent decrease in dentin. The softening of the tissue due to decrease in hardness is a common factor among tooth to lose its integrity and enable cavity formation in the vicinity of exposed area $^{24}$. In either case, the oral environment becomes feasible to develop caries. ${ }^{30-32}$

\section{Compression Test}

Compression test is the most widely used technique, which is used to determine the compressive force or crush resistance force of a material and also its ability to recover at a specific interval of time. These tests are most commonly used to understand the behavior of the material/substance under different loads. The maximum stress that a material can withstand over a particular time due to a specific load applied will be tested. Compression test can be performed to measure Load, Deflection and Work at Rupture, Maximum Load, Deflection and work at M aximum Load, Stiffness, Chord Slope, Offset Yield, Stress and Strain. The behavior of load, Stress and strain is applicable to define the mechanical properties of teeth based on its strength. Similarly researchers have studied the properties of teeth using compressive strength analysis. Initially the teeth have to be sectioned to obtain separate Enamel and Dentin portions which will be processed and then exposed to the testing machine against a specific load. It is proclaimed that Enamel is more brittle than Dentin, as concluded by its rupturing ability by the values of Stress and strain. But it is found to be harder than Dentin. Similar studies should be performed on radiated teeth to understand its level of decrease and increase in stress or strain. $^{33}$

\section{Indentation tests}

In normal traditional indentation techniques the load is high and has large indentation area. But this can be one of the limitations for the hardness evaluation due to the 
presence of microstructural constituents. Therefore, in recent years, nanoindentation techniques have been introduced which has a load range of $0.1-5000 \mathrm{mg}$ with a minimum indentation area of lum size. This is one of the recommended techniques that could be used in testing microhardness of the material whose strength varies with different areas of interest. Depending on the indentation size and type, different variables such as applied force, displacement into the material surface, and load time could be measured by a nanoindenter. These measurements are useful in driving the hardness, modulus of elasticity, and other mechanical properties of teeth. The predicted hardness and modulus of elasticity for human enamel is known to be $5 \mathrm{GPa}$ and $70 \mathrm{GPa}$ respectively. Many such studies have been done to characterize the enamel properties by analyzing its indentation behavior. Hence it could be one of the techniques to measure the variation in tooth Enamel caused due to radiation interactions. ${ }^{34.35}$

\section{Average Enamel Thickness}

The Enamel thickness has a great significance on the human permanent teeth in preventing caries formation. Increased enamel thickness is attributed to greater bite force or due to smaller crown size. Thus, assessing the differences in morphology of enamel thickness can help in comprhending the properties of teeth. Average Enamel Thickness (AET) is one such criterion which has to be related in interpreting the thickness variability. The thin sections of the teeth should be produced using standard histological procedures to examine AET. Each thin enamel sections should be mounted on a separate microscope slides and graded. Further the sections will be polished with an aluminum oxide powder in an ultrasonic bath to remove any exudate or debris particle. This will be followed with alcohol dehydration and mounted using Xylene placing the cover slip. The observation will be carried out under a high-powered microscope and data obtained is used to calculate Average Enamel thickness.

One of the studies has assessed enamel thickness along the entire human deciduous tooth row and it was concluded that M ean AET had increased way through the row. M olar enamel thickness was greater due to bite forces frequently applied and hence this can be one of the parameters to study changes in enamel variation/ degradation. ${ }^{36}$

\section{Techniques to analyze morphological changes of teeth after radiation therapy}

Radiation causes morphological changes in the tooth structure, which are visible under Microscopic analysis. Some of the techniques involved in evaluation are SEM (Scanning Electron Microscopy), OCT (Optical Coherence Tomography) and confocal laser scanning M icroscopy.

\section{Scanning Electron M icroscopy Analysis}

The Scanning Electron Microscope (SEM) analysis is very suitable technique for surface morphology research. The SEM uses a focused beam of high-energy electrons to generate a variety of signals at the surface of solid specimens. These high energy electron particles produce signals that are generated during the interaction of secondary and reflected electrons with the sample. The signals give information on the external morphology (texture), chemical composition, crystalline structure and orientation of subjected sample materials. The data obtained is from a selected area on the surface of the sample, which gives a 2-dimensional image that displays internal structure of the tissues. To avoid any eventual atmospheric effects, those analysis are made in a devoid of air space. ${ }^{37-38}$

The studies performed using SEM to determine the changes in the enamel structure of the non-irradiated and irradiated teeth had showed clear observable distinctions. The cross sectional study of teeth control samples display well-organized prisms, surrounded by interprismatic regions. But the electron micrographs of irradiated groups with a dosage rate of $30 \mathrm{~Gy}-60 \mathrm{~Gy}$ has revealed a progressive change in the enamel surface. With increasing doses of irradiation, a progressive change was also observed in the prismatic structure of the enamel, thus making difficult in identification of the prisms. In addition to these feature radiation exposure to $60 \mathrm{~Gy}$ and above has shown amorphous surface, and the visualization of the prisms and hydroxyapatite crystals are hard to define thus 
losing the definition of the interprismatic space ${ }^{.36-39}$

The microscopic studies will give a clear distinction of structural variation in organic and inorganic compounds. Hence SEM being a highly sensitive method, with magnification range between $20 \mathrm{X}-30,000 \mathrm{X}$ can be a useful technique for imaging studies.

\section{Optical Coherence Tomography (OCT) Analysis}

Optical coherence tomography is generally used to visualize the morphological characteristics of the lesions. But certain studies have used OCT to detect alterations of the tissues optical properties and for qualitative analysis of the carious lesions at the enamel-dentin junction. The images of OCT are based on the optical properties of the sample and they are made using false color mapping, where the white color corresponds to a high spreading coefficient equivalent to demineralized areas and the black color corresponds to a low spreading coefficient. ${ }^{40}$

The studies dealing with irradiated teeth showed the images of demineralised area due to the cariogenic challenge on enamel and dentin and of the enamel-dentin. The healthy dentin exhibits a whitish stripe that will be less homogeneous than that of the enamel. The tooth structures with carious lesions shows a whitish stripe deeper than did the healthy teeth. Hence it is concluded that radiation treatment of sound teeth might cause deeper carious lesions than in non-irradiated teeth been subjected cariogenic challenge. ${ }^{40}$

Though the OCT technique is not been extensively used, the research studying remineralisation or demineralization of the teeth will have profound results.

\section{Confocal Laser Scanning M icroscopy}

Dentin morphology found in dental caries has been studied for many years. Initially it was observed under optical microscopy, and later using electron microscopy. Confocal laser scanning microscopy (CLSM) used with several fluorescent dyes such as alizarin red is studied to see normal dentinal tubules. But the CLSM studies on dentinal tubules in human caries are very rare. ${ }^{41-42}$ The principle of the confocal microscope is based on the reflected light microscopy that optically scans a specimen by sending and receiving light through pinholes of variable apertures. The tooth has to be placed on a slide with plasticine with the tip of the cusp perpendicular to the lens with immersion oil and a cover slip. A second drop of oil has to be placed between the cover slip and the objective lens for the better magnification of the image. ${ }^{43}$

Confocal microscopy is useful for studying the tooth surfaces, particularly molar and premolar as it can only be used for those macro surfaces that do not have undercuts. Fully 3-D rendered models can be created of much smaller objects. The benefit of this method is that the higher resolution images can be obtained compared to many digital scanning devices. ${ }^{44}$ The microorganisms on the enamel plaque can be identified under observation. ${ }^{41}$ It is a nondestructive sensitive technique in demonstrating mineral changes in carious dentin. The area of demineralized dentin in translucent zone appears to be dark. Deep carious dentin has hyper-mineralized areas advancing towards the de-mineralized zone. The caries size and its rate of penetration results in the formation of dentinal sclerosis receives a transparent and glass-like appearance. ${ }^{42}$ Hence Visualization of the dentinal tissues by microscopic analysis is an important tool to determine the extent of damage occurred by hazarding substances.

These techniques will analyze the changes occurring in tissue due to imbalance conditions. Whereas maintenance of healthy oral environment will prevent in the development of caries among HNC patients. The Oral care guidelines that should be followed under clinical practice have been mentioned in this paper. Also to perform studies related to oral cavity, the samples to be tested should be maintained in good oral condition to maintain the healthy environment.

\section{Tissue storage media}

To maintain the oral biological environment during in vitro testing, a suitable media that ensures oral cavity is recommended. For this purpose various medias have been used by researchers, such as distilled water, artificial saliva $^{50}$, saline, Hanks Balanced salt solution ${ }^{51}$, Phosphate 
Buffer saline, water, milk ${ }^{52}$ etc. Each media has its own effect on the efficiency and limitations. The properties of the teeth can also differ based on these media. Artificial Saliva and HBSS is known to maintain the elastic properties of the tissue whereas saline has shown to decrease in acoustic properties. ${ }^{53}$ Currently there is no single solution that satisfy and meet the conditions of oral environment for temporary storage of the extracted teeth. Hence further research is required in thisfield.

\section{Oral Care strategy}

The Guidelines published by Multinational Association of Supportive Care in Cancer (MASCC), ${ }^{45}$ National Comprehensive Cancer Network ${ }^{46}$ and Cochrane Review ${ }^{47}$ recommends Standard Basic Oral care to be practiced to prevent infections and reduce oral cavity issues developing in Chemo-radiated patients. The important Oral health care regimen is as follows: $:^{48-49}$

- Dental specialists have to pre-evaluate patients for restorations or extractions undergoing radiotherapy.

- Maintaining good oral hygiene during and after radiation will reduce the infections and dental complications. It involves brushing teeth using soft bristles in a non-traumatic fashion, rinsing frequently using solutions such as normal saline with sodium bicarbonate. Application of moisturizing agents and use of daily dental fluorides is mandatory.

- Periodic dental evaluation and cleaning of oral cavity also servesthe mission.

\section{References}

1. M oore S, Burke MC, Fenlon MR, Banerjee A. The role of the general dental practitioner in managing the oral care of head and neck oncology patients. Dent Update. December 2012; 39(10):694-702.

2. Thariat J et al. Compliance with fluoride custom trays in irradiated head and neck cancer patients. Support Care Cancer. August 2012; 20(8):1811-4.

3. Aguiar GP et al. A review of the biological and clinical aspects of radiation caries. J Contemp Dent Pract. July 2009; 10(4):83-9.

4. Jham BC and Freire ARS. Oral complications of radiotherapy in the head and neck. BrazJ Otorhinolaryngol. Sept- Oct 2006; 72(5):704-8.

5. M eurman JH and Grönroos L. Oral and dental health care of oral cancer patients: hyposalivation, caries and infections. Oral Oncol. June 2010; 46(6):464-7.

6. Lawrence TS, Ten Haken RK, Giaccia A. Principles of Radiation Oncology. In: DeVita VT Jr., Lawrence TS, Rosenberg SA, editors. Cancer: Principles and Practice of Oncology. Philadelphia: Lippincott

\section{Conclusion}

Radiotherapy is a therapeutic treatment employed towards killing of cancerous cells. It is combined with chemotherapy in a current treatment care regimen. The common side effects include the conditions such as Oral Mucositis, Xerostomia, candidiasis, osteoradionecrosis, soft tissue necrosis, dry mouth and radiation caries. The effects of chemo-radiation therapy have been studied extensively and this paper gives an explanation on techniques to analyze the effects of radiation on tooth. The side effects of radiation therapy on Enamel and Dentin could be analyzed by evaluating the mechanical properties of the teeth before and after radiation therapy using micro hardness test, Indentation tests, and compression strength analysis at different regions of Enamel and Dentin. These results can be further supported by morphological changes in the Enamel and Dentin using Scanning Electron Microscopy, Optical Coherent Tomography and Confocal Laser M icroscopy. The morphological studies support the fact that variations in hardness are solely dependent on variations in tissue structure. And it can be prevented by providing favorable oral environment for 6 months period of pre and post radiation treatment which helps in enhancing the annealing effect of organic and inorganic tissues on enamel and dentin ${ }^{54}$. Further this article concludes that there is a scope for future research on enhancing the annealing effect of Enamel and Dentinal tissues; thereby help in the prevention of radiation caries

Williams and Wilkins, 2008; $8^{\text {th }}$ edition

7. Stevenson M M. Head and Neck Cancer Treatment protocols. Anand J, Braden CD, Harris JE Editors. Updated May 31 2013: Medscape Available at : http://emedicine.medscape.com/article/2006216overview

8. The Oral Cancer Foundation. Information - Support - Advocacy Research... and Hope. Last modified April 2014. Available at: http:// www.oralcancerfoundation.org/dental/dentalcomplications.htm

9. Trotti A, Bellm LA, Epstein JB, et al. Mucositis incidence, severity and associated outcomes in patients with head and neck cancer receiving radiotherapy with or without chemotherapy: a systematic literature review. Radiother Oncol. 2003;66:253-262.

10. Dirix P, Nuyts S, Van den Bogaert W. Radiation induced xerostomia in patients with head and neck cancer: a literature review. Cancer 2006;107:2525- 2534. 
11. Semba SE, M ealey BL, Hallmon WW. The head and neck radiotherapy patient: Part 1 - oral manifestations of radiation therapy. Compend Contin Educ Dent 1994;15:252-260

12. Fajardo LF, Bertrong M, Anderson RE. Salivary glands In: Fajardo LF, Bertrong $M$, Anderson RE, editors. Radiation pathology. M innesota: Oxford University Press, 2001;265-270

13. Chao KS, Deasy JO, Markmon J, et al. A prospective study of salivary function sparing in patients with head-and-neck cancers receiving intensity-modulated or three-dimensional radiation therapy: initial results. Int J Radiat Oncol Biol Phys. 2001; 49:907-916.

14. Roesink JM, M oerland MA, Battermann JJ, et al. Quantitative dosevolume response analysis of changes in parotid gland function after radiotherapy in the head-and-neck region. Int J Radiat Oncol Biol Phys. 2001;51:938-946.

15. Nanci A. Ten Cate's Oral Histology: Development, Structure, and Function. 6th ed. St Louis, M O: M osby Elsevier Science. 2007

16. Gwinnett AJ. Structure and composition of enamel. Operative Dentistry 1992; (Suppl 5):10-17.

17. Dominique Brooks. Effects of Radiation on Teeth. Last updated 28 Jan 2015. Live strong Foundation Available at: http:// www.livestrong.com /article/248428-effects-of-radiation-on-teeth/

18. Lieshout HF and Bots CP: The effect of radiotherapy on dental hard tissue-a systematic review. Clin Oral Investig. 2014; 18(1):17-24.

19. Al-Nawas B, Grötz KA, Rose E, et al. Using ultrasound transmission velocity to analyse the mechanical properties of teeth after in vitro, in situ, and in vivo irradiation. Clin Oral Investig. 2000; 4(3):168-72.

20. Kielbassa AM , Hinkelbein W, Hellwig E, et al. Radiation-related damage to dentition. Lancet Oncol. 2006; 7(4):326-35

21. Silva AR, Alves FA, Antunes A, et al. Patterns of demineralization and dentin reactions in radiation-related caries. Caries Res. 2009; 43(1):43-9.

22. Markitziu A, Gedalia I, Rajstein J, et al. In vitro irradiation effects on hardness and solubility of human enamel and dentin pretreated with fluoride. Clinical Prevent Dent. 1986; 8(4):4-7.

23. Pioch T, Golfels D, Staehle HJ. An experimental study of the stability of irradiated teeth in the region of the dentinoenamel junction. Endod Dent Traumatol. 1992; 8(6):241-4.

24. Grötz KA, Duschner H, Kutzner J, et al. New evidence for the etiology of so-called radiation caries. Proof for directed radiogenic damage on the enamel-dentin junction. Strahlenther Onkol. 1997; 173(12):668-76.

25. Soares C], Castro CG, Neiva NA, et al. Effect of gamma irradiation on ultimate tensile strength of enamel and dentin. J Dent Res. 2010; 89(2):159-64

26. Jham BC, Freire ARS. Oral complications of radiotherapy in the head and neck. Rev Bras Othorrinolaringol. 2006; 72 (5): 704-8.

27. M jor IA. Human coronal dentine: structure and reactions. Oral Surg Oral M ed Oral Pathol 1972; 33:810-823

28. Cheung DT, Perelman N, Tong D, Nimni ME. The effect of gammairradiation on collagen molecules, isolated alpha-chains, and crosslinked native fibers. J Biomed M ater Res. 1990; 24:581-589

29. Pioch T, Golfels D, Staehle HJ. An experimental study of the stability of irradiated teeth in the region of the dentinoenamel junction. Endod Dent Traumatol. 1992; 8:241-244

30. Goncalves L M N et al. Radiation therapy alters microhardness and microstructure of enamel and dentin of permanent human teeth. Journal dentistry. 2014; 42:986-992.

31. Markitziu A, Gedalia I, Rajstein J, et al. In vitro irradiation effects on hardness and solubility of human enamel and dentin pretreated with fluoride. Clinical Prevent Dent. 1986; 8(4):4-7.

32. Wang $L$ et al. Mechanical properties of dental restorative materials: relative contribution of laboratory tests. J Appl Oral Sci. 2003; 11(3): $162-7$

33. Chun KJ, Choi HH, and Lee JY. Comparison of mechanical property and role between enamel and dentin in the human teeth. Journal of Dental Biomechanics. 2013:

34. Lim CT, Omar BHB and GohJCJ. Nanoindentation study of normal and osteoporotic bones.
35. HalgasR, Dusuza J, Kaiferova J, Kovacsova L, Markovska N. Nanoindentation testing of Human Enamel and Dentin. Ceramics Silikáty. 2013;57 (2):92-99

36. Mahoney P. Testing functional and morphological interpretations of enamel thickness along the deciduous tooth row in human children. American Journal of Physical Anthropology. 2013; 151(4):518-525

37. Azinovic et al. SEM Analysis of Tooth Enamel. Coll. Antropol. 2003;1: 381-386

38. Susan Swapp. Scanning Electron Microscopy (SEM). Page: Geochemical Instrumentation and Analysis. Last Modified: 15 June 2015; Science education research Center Carlton college Available at: http://serc.carleton.edu/research_education/geochemsheets/techn iques/SEM.html

39. M ellara et al. The effect of radiation therapy on the mechanical and morphological properties of the enamel and dentin of deciduous teeth-An in vitro study. Radiation Oncology. 2014; 9:30

40. Lee $D P$, et al. The effects of ionizing radiation on the development of human caries lesions in vitro. Clin Lab Res Den. 2014; 20 (1): 46-53

41. Al-Salihi KA and Tarmidzi NABA. Preliminary Study of In Vivo Formed Dental Plaque Using Confocal Microscopy and Scanning Electron Microscopy. Journal of Dentistry, Tehran University of Medical Sciences. 2009; 6(4):159-167

42. Marzuki A F and Masudi SM. Confocal laser scanning microscopy study of dentinal tubules in dental caries stained with alizarin red. Archives of Orofacial Sciences. 2008; 3(1): 2-6

43. Haydenblir R, Benjamin P Z and Smithl P. Evolution of Human Enamel Growth Analyzed by Confocal M icroscopy. 228-235

44. Evans A R, Harper I S and Sanson GD. Confocal imaging, visualization and 3-D surface measurement of small mammalian teeth. Journal of Microscopy, Nov 2001; 204(2):108-119

45. Keefe DM, Schubert MM, Etting LS, et al. Updated Clinical Practice guidelines for the prevention and treatment of mucositis. Cancer. 2007; 109: 820-839

46. Bensinger W, Schunert M M, Ang KK, et al. NCCN task Force Report. Prevention and $M$ anagement of M ucositis in Cancer Care. J Natl compr Canc Netw. 2008;6: (Suppl 1)S1-S21

47. Worthington HV, Clarkson JE, Eden OB. Interventions for preventing factor mouthwashes heal oral ulcers during Head and Neck Radiotherapy. Int J Radiat Oncol Biol Phys, 1998;41:747-754

48. M c Guire DB, Correa ME, Johnson J, et al. The role of Basic Oral care and good Clinical Practice Principles in the Management of oral mucositis. Support Care Cancer, 2006; 14: 541-547.

49. National Cancer Institute, Oral Complications of Chemotherapy and Head/Neck radiation (PDQ). Accessed Oct 9, 2008. Available at http://www.cancer.gov/cancertopics/pdg/supportivecare/oralcompl ications.

50. Bajaj N, Grewal N, Grewal S, Monga P. Association of physical properties and maintenance of sterility of primary teeth in human tooth bank. Journal of Indian Society of Pedodontics and Preventive Dentistry. 2014; 32(4): 279-285

51. Grewal N, Kaur M. Evaluation of the effect of storage on sterilization and structural integrity of enamel of teeth preserved in a tooth tissue bank - An in-vitro study. Thesis submitted in partial fulfillment of the requirements for the degree of M DS of Baba. Faridkot: Farid University of Health Sciences. 2006

52. Roberto P W, Sonoda CK, Martins CM, Melo ME, Pellizzer EP, Mendonça MR, Panzarini SR. Storage Media For Avulsed Teeth: A Literature Review. Brazilian Dental J ournal. 2013; 24(5): 437-445

53. Rauma K, Kempfa K, Hans Heina J, Schubertb J, Maurerb P. Preservation of microelastic properties of dentin and tooth enamel in vitro-A scanning acoustic microscopy study. Dental materials. 2007; 23: $1221-1228$

54. Hegde M N, Hegde ND, KumariSN, Sanjeev G, Priya. Annealing effect of irradiated tooth using surface micro hardness testing- an invitro study.WJPR, 2015;4(12): 1149-1160 